\title{
On the Foundation of the Philosophy of Information
}

\author{
Luciano L Floridi \\ Departments of Philosophy and Computer Science \\ University of Oxford \\ Wolfson College, Oxford, OX2 6UD, UK \\ luciano.floridi@philosophy.oxford.ac.uk
}

\begin{abstract}
Philosophers have recently begun to address the new intellectual challenges arising from the world of information and the information society. Consequently, a new and vitally important area of research has begun to emerge, the philosophy of information (PI). This paper is the first attempt to analyse the potential nature of PI systematically. The paper aims to explain (1) what PI is (2) how PI has emerged (3) why there should be a new philosophical discipline such as PI, (4) what PI's scientific goals are and, finally, (5) what interpretations of PI are possible, either as a philosophy of science/technology or as a philosophy of information design. It is argued that PI is the philosophy for the information age, as it offers the systematic treatment of the philosophical foundations of the world of information and of an information society.
\end{abstract}

Keywords: Information and Communication Technology, Information Science, Information Society, Informationtheoretic Methodology, Infosphere, Philosophical Innovation, Philosophy of Information, Philosophy of Information Design, Philosophy of Science, Semanticisation, Unified Theory of Information.

\section{INTRODUCTION}

The information society

The information society has been brought about by the fastest growing technology in history. No previous generation has ever been exposed to such an extraordinary acceleration of technological power and corresponding social changes. Total pervasiveness and high power have raised ICT (information and communication technology) to the status of the characteristic technology of our time, both rhetorically and iconographically. The computer presents itself as a culturally defining technology and has become a symbol of the new millennium, playing a cultural role comparable to that of mills in the Middle Ages, mechanical clocks in the seventeenth century and the loom or the steam engine in the age of the industrial revolution.

These profound transformations have been already interpreted in terms of a digital-information turn. ICS (the cluster of Information and Computing Sciences that deal with the world of data structures and flows, including systems theory) and ICT applications are nowadays the most strategic of all the factors governing the life of our society and its future. The most developed post-industrial societies literally live by information, and ICS-ICT is what keeps them constantly oxygenated.

Three Relations between Philosophy and Computing Once it is acknowledged that the development of human society is at a stage in which issues concerning the creation, dynamics, management and utilisation of information are vital and very rich in consequences, it becomes legitimate to ask how far this macrocultural phenomenon concerns philosophy, both as an intellectual area, itself deeply affected by the recent transformations, and as a discipline that can provide the conceptual means necessary to explain and understand such significant phenomena.

For the sake of simplicity, the different relations between philosophy, ICS and ICT can be organised into three, sometimes overlapping, areas (see [1] for further details).

\section{Philosophy Computing}

This is the branch of Humanities Computing that studies the applicability, and hence the development of ICT solutions to philosophy and logic, for teaching and informationmanagement purposes (e.g. didactic software and electronic editions of texts, or databases). In such a context, wondering what is implicit in the relation between computing, ICT and philosophy means to ask not only whether philosophers may be able to exploit recent technological innovations for their work, and if so, to what extent, but also what it means for a philosopher to be abreast of the technology of our time. To give a specific example, until recently, the American Philosophical Association Committee for Philosophy and Computers focused almost entirely on what has been defined here Philosophy Computing. The most recent example of a Philosophy Computing product is probably the Routledge Encyclopedia of Philosophy on CDROM.

\section{Digital Philosophy}

This is a more recent development in the field, namely the use of ICT in philosophical investigations to model conceptual problems, as in the case of Artificial Morality, the use of computerised models for the study of rational choice in ethical contexts.

It is clear that both (1) and (2) look at what ICT can do for philosophy. They do not investigate what philosophy can do for ICS. We shall see that this is one of the four goals of 3. The Philosophy of Information (PI).

\section{THE DEFINITION OF PI}

What is PI precisely? A first step towards a clarification of the nature of PI can be taken by comparing it to other special branches of philosophy, such as the philosophy of mathematics or the philosophy of logic. These are old philosophical subjects, which nevertheless have acquired their salient features and become autonomous fields of investigation only relatively late in the history of thought. A second step requires 
the introduction of two distinctions.

1) All "philosophies of" manifest a tendency to converge towards two poles, one phenomenological and the other metatheoretical. The philosophy of language and the philosophy of knowledge are two examples of "phenomenologies" or philosophies of a phenomenon. Their subjects are semiotic codes, not linguistic theories; and epistemic capacities, practices and contents, not cognitive sciences. The philosophy of physics and the philosophy of social sciences, on the other hand, are obvious instances of "metatheories". They investigate problems arising from organised systems of knowledge, which only in their turn investigate natural or human phenomena.

2) Now, many "philosophies of" show only a tension towards the two poles, often combining phenomenological and metatheoretical interests. This is the case with the philosophy of mathematics and the philosophy of logic, for example, and the philosophy of information itself. These philosophies show a tendency to work on specific classes of first-order phenomena, but they also address these phenomena working their way through second-order approaches and issues, that is by starting from a metatheoretical interest in specific classes of theoretical statements, methodologies, technical concepts etc. concerning those very same classes of phenomena.

The tension pulls each specific "philosophy of" towards one or the other pole. The philosophy of logic, to rely on the previous example, is metatheoretically biased. It shows a constant tendency to concentrate mainly on problems arising from logic understood as a specific mathematical theory of deductive inferences formally valid, whereas it pays less attention to problems concerning logic as a field of phenomena, what one may call, for want of a better description, the expression of human rationality. Vice versa, the philosophy of information is phenomenologically biased. It is primarily concerned with the whole domain of phenomena represented by the world of information (infosphere) and the information society, and can be seen to incline towards a metatheoretical approach only in so far as it may address its problems by starting from the vantage point represented by ICS. As we shall see, this explains the tension between (a) considering PI as separate from, but providing the methodological foundation of further information-theoretic approaches in philosophy and (b) considering the latter as cases of PI, belonging to it as specific branches.

The following definition attempts to capture the clarifications introduced so far:

(PI) the Philosophy of Information is the descriptive as well as normative branch of philosophy primarily concerned with the conceptual and foundational investigation into the nature of information, its dynamics and utilisation.

A few explanatory comments may be in order. By "dynamics of information" the definition refers to (a) the constitution of information environments, with their systemic properties, interactions, internal developments etc. and (b) information life cycles, i.e. the series of various stages in form and functional activity through which information can pass, from its initial occurrence to its final utilisation and possible disappearance. ${ }^{1}$

\footnotetext{
${ }^{1}$ For example, a typical life cycle can include the following phases: occurring (discovering, designing, authoring, etc.), processing and managing (collecting, validating, modifying, organising, indexing, classifying, filtering, updating, sorting,
}

The meaning of "descriptive", "normative", "conceptual" and "foundational" as used in (PI), although standard in the philosophical lexicon, will be fully clear once the goals of PI are introduced. To anticipate: PI treats information as something more elementary and basic than meaning or knowledge, on the same level with being. It provides a unified, explanatory theory of what information is, not a quantitative theory of data communication. And from an environmental perspective, it legislates on what may count as information and how information should be adequately created and manipulated. PI is based on a philosophy of the history of thought, which sees it as the most recent stage in the development of Western philosophy. If the scientific revolution in the seventeenth century made philosophers redirect their attention from the nature of the knowable object to the epistemic relation between it and the knowing subject, the subsequent growth of the information society and the appearance of the infosphere, as the true environment in which millions of people spend their time nowadays, have led contemporary philosophy to privilege critical reflection first on the domain represented by the memory and languages of organised knowledge, then on the instruments whereby the infosphere is managed and finally on the nature of its very fabric and essence, information itself.

\section{THE EMERGENCE OF PI}

Is time ripe for the elaboration of a cohesive theoretical framework for PI? So far we have seen two different lines of argument in favour of an affirmative answer:

1) scientific, technological, and sociological considerations, such as the development of ICS and ICT; the arising of the information society and of the knowledge economy, etc., and

2) the development of the history of philosophy, whose central focus has moved from being, to knowledge, to meaning, to information.

A third line of argument, perhaps more interesting here, is provided by the development of contemporary philosophy interpreted as a conceptual system:

3) PI has emerged because of philosophy's present scholasticism (see below for a detailed definition) and hence its distance from contemporary issues, as an alternative approach interested in, and capable to deal with the infosphere, which, in a way only apparently paradoxical, has been brought about by past philosophy.

To understand (3), a fundamental dynamics in the development of reflection needs to be briefly explained.

In order to flourish, the mind must make sense of its environment. It does so by investing reality with meaning. This "semanticisation of being" consists in the development of environmentally constrained epistemic narratives (self, ordinary experience, science...). It is a process that has an ideal direction, which can be called "windowing", resulting from three conceptual vectors: externalisation of the conceptual environment within which the mind lives (from infrasubjective to inter-subjective), reification (hypostatisation) of

storing, networking, distributing, accessing, retrieving, transmitting etc.) and using (monitoring, modelling, analysing, explaining, planning, forecasting, decision-making, instructing, educating, learning, etc.). 
this environment, and de-physicalisation of nature (framing or virtualising the world). The information society can be seen as the most recent stage in this windowing process. Now, in the course of its evolution, the process of semanticisation leads to a fixation of the constructive conceptualisation of reality (views of the world), which ends into a (scholastic) closure of the system. This "scholastic loop" is the inevitable destiny of reflection: reflection is doomed to be initially innovative, to establish itself as a specific dominating paradigm (as the specific philosophy in power), hence to become fixed and increasingly rigid, further reinforcing itself, until it finally becomes intolerant towards alternative conceptual innovations and so incapable of dealing with the ever changing environment (cf. Kuhn's concept of "normalisation" in [2]). The old paradigm is finally challenged and replaced by further, innovative reflection only when the latter is sufficiently robust to represent a more viable alternative. Three factors can then force a conceptual system to innovate. There is the internal force, which pushes the system from within towards the threshold of change. This is the system's natural inertia and a certain degree of positive resistance against innovation, which causes the "scholastic loop" just seen, and hence a constant increase in the fragility of the system. And there are two external forces, which pull the system from without. They are the substantial, conceptual novelties in the environment of the system and the appearance of a new approach capable to deal with them more successfully.

\section{The Internal Force: Scholasticism}

Although this paper does not concern the internal crisis undergone by the philosophy system, it is important to keep in mind that contemporary philosophy may benefit from a radical reflection on its present state and tasks. Whenever philosophers become involved only with their own issues, while questions that would be of lively interest outside the academia are disregarded as irrelevant, the phenomenon may be defined as scholasticism, the pedantic and intolerant adherence to the traditional teachings, "right" methods, rigid canon of "important" texts or selection of "truly philosophical" problems set by of a particular school of thought. Whenever reflection undergoes a process of scholasticisation, an increasingly wider gap between philosophers' problems and philosophical problems begins to grow. As a consequence, philosophy becomes less and less relevant, interesting and useful: it addresses questions only philosophers belonging to the specific school are keen to ask, while failing to help us to understand, improve, control, or live morally in our world. It is not unusual to hear the complaint that society does not listen to philosophers anymore, but it seems fair to retort that maybe it is the kind of philosophical research that our graduates are invited to develop that is no longer engaging the real world.

Scholasticism is the negative pushing force of innovation, internal to any philosophical system. It fossilises thought, and by making a philosophical school increasingly rigid and less responsive, it weakens its capacities of reaction to external inputs, divorces it from reality, and hence prepares the ground for a solution of the crisis.

\section{The External Forces: PI and the Infosphere}

We are able to classify philosophy's present predicament as one of scholasticism because we perceive that the system has become conceptually inadequate to cope with the new world surrounding it and hence needs to be innovated. Scholasticism, however, represent only a necessary condition of innovation.
For it can only indicate that philosophy has reached a stage when it needs to develop a new approach, or a new methodology, or a new frame of concepts and theoretical concerns. It does not suffice to indicate which direction the innovation should take. Historically, this is the task of other forces. Since the separation of the various forms of knowledge from philosophy itself - and this begins already in the Middle Age, with the separation between Theology and Philosophythe pulling force has become necessarily external to philosophy (this is the heteronomous nature of philosophical innovation). In the past, it has been represented by factors such as Christian theology, the scientific revolution, the development of human sciences, the discovery of non-Euclidean geometries, the foundational crisis in mathematics, the establishment of mathematical logic, evolutionary theory, psychoanalysis, the theory of relativity, quantum physics. Nowadays, this pulling force is represented by the complex world of information and communication phenomena, their corresponding sciences and technologies and the new environments and social life brought about by them.

Let us reconsider now our initial question: is time ripe for the elaboration of a cohesive theoretical framework for PI? It seems that the answer must be affirmative because the world is moving towards PI, the history of philosophy is moving towards PI, and the dynamic forces regulating the development of the philosophical system are moving towards PI. But then, what kind of PI should we try to develop? An answer to this question presupposes a clearer view of PI's possible goals.

\section{THE FOUR GOALS OF PI}

On the basis of the previous analysis, PI emerges as a new philosophical paradigm with four main goals. PI can be expected to develop:

\section{a) a Unified Theory of Information (UTI) (Classic Goal)}

UTI constitutes the kernel of PI: it analyses, explains and evaluates the nature of information, its dynamics and its utilisation, with special attention to systemic issues arising from information environments. This is a classic goal insofar as it addresses the typical philosophical question "what is information?".

\section{b) an Information-theoretic Philosophical Method (IPM)} (Innovative Goal)

On the basis of (a), PI can be expected to develop informationtheoretic and computing-based concepts, methods, tools and techniques, to model, explain and solve both traditional and new types of philosophical problems, arising in the infosphere and in the information society. It is important to stress that IPMs have already been applied in many philosophical areas, to extend our understanding of

- the cognitive and linguistic abilities of humans and animals and the possibility of artificial forms of intelligence (the Philosophy of AI; Informationtheoretic Semantics; Information-Theoretic Epistemology)

- inferential and computational processes (the Philosophy of Computing; the Philosophy of Computer Science; Information-flow Logic)

- the organizational principles of life and agency (the Philosophy of Artificial Life; Cybernetics and the 
Philosophy of Automata; Decision and Game Theory)

- new approaches to modelling physical and conceptual systems (Formal Ontology, Theory of Information Systems)

- the methodology of scientific knowledge (Modelbased Philosophy of Science; Computational Methodologies in the Philosophy of Science)

- $\quad$ ethical problems (Computer and Information Ethics)

- aesthetical issues (Digital Multimedia/Hypermedia Theory, Hypertext Theory and Literary Criticism)

- $\quad$ psychological, anthropological and social phenomena characterising the information society and human behaviour in digital environments (Cyberphilosophy).

Indeed, the overlap between these Proto-Philosophies of Information and many other areas of philosophy shows that the development of the latter can be divided into pre- and postinformation turn.

\section{c) a Systematic Foundation (Systemic Goal) of Proto-PI}

On the basis of (b), PI can be expected to provide a unified and cohesive, theoretical framework for the various branches of the philosophy of information and computing (the proto-PI listed above), from the Philosophy of AI to Computer Ethics.

\section{d) a Special Philosophy of Science/Technology (Methodological Goal)}

Still on the basis of (b), PI can also be expected to provide a unified and cohesive, theoretical framework for the information sciences and related disciplines, by isolating and systematising critically their conceptual, methodological and theoretical basis.

Once PI is correctly understood as the study on the philosophical issues arising from the infosphere and the information society, and as a new philosophical methodology that can provide the foundation of both ICS and Proto-PI, it becomes easy to see why it cannot possibly be misunderstood for a sort of applied ICT or an ICT made simple - the role of mediation between the expert technician and the potential user still uninformed is certainly important, but completely irrelevant in this context-or for a more general and nonempirical (and therefore useless, from the practical view represented by the market) continuation of ICS research by other means, to say it with von Clausewitz, as if by philosophy of information one should mean "abstract and a priori ICS".

The interpretation of the philosophy of information as the philosophy of the infosphere also clarifies the rationale behind some inclusions and exclusions, otherwise quite surprising. On the one hand, we realise that the Proto-PI, like the philosophy of AI for example, do not belong, strictly speaking, to the philosophy of information as its branches, stemming from a common source, but rather as its fields of application. That in both cases people are working on a common ground represented by ICS and ICT is irrelevant and misleading: the philosophy of AI is above all a metatheoretical philosophy, a philosophy of the theory, whose primary task for a long time has been that, eminently anthropocentric, of understanding better the human mind by studying forms of non-biological intelligence, a program of research which obviously bears too faint a relation with the infosphere to be a constitutive part of PI. On the other hand, an excessive concern with the metatheoretical aspects of the philosophy of information may lead one to miss the important fact that it is perfectly legitimate to speak of PI even in authors who lived centuries before the information revolution, and hence that it will be extremely fruitful to develop a historical approach and trace PI's diachronic evolution, as long as the technical and conceptual framework of ICS are not anachronistically applied but are used to provide only the conceptual method and privileged perspective to evaluate in full reflections developed on the nature, dynamics and utilisation of information before the information digital revolution (three clear examples are provided by Plato's Phaedrus, Descartes' Meditations and Nietzsche's On the Use and Disadvantage of History for Life). This is significantly comparable with the development undergone by the philosophy of mathematics, which established itself as an academic discipline only very recently in the history of thought, after the foundationalist crisis, but that is certainly present in many past philosophers, even if sometimes couched in different cloths. Similar considerations can finally be extended to the distinction between computer art, which is certainly not a subject of investigation of PI just because computers are being employed as expressive means, and conceptual art, which even in extreme forms of expression, such as land art, indubitably represent an aesthetical valorisation of the constructed world of information, whose poetics must be placed within the more ample context of a philosophy of the infosphere to be properly understood.

\section{SHOULD THERE BE A PI?}

So should there be a PI, and if so, what kind of PI is more likely to be developed? It has been argued above that any negative answer to the former question is inconsistent with the development of (a) contemporary society, (b) the history of philosophy and (c) our present philosophical system. Affirmative answers, on the other hand, can lead to a minimalist or a maximalist approach.

\section{Minimalist Approach}

The Systemic and Methodological Goals (c)-(d) lead to a minimalist approach: there should be a philosophy of information, but this should be understood merely as a foundational philosophy of science/technology, whose theoretical input is provided by (i) various informationtheoretic and computing-based philosophies and (ii) ICS.

The problem with the minimalist approach is that, although it may appear to be theoretically safer because more modest, it fails to take into account the fact that (c) and (d) require UTI and IPM. Either there is no PI, or a minimalist approach to PI leads inevitably to a maximalist approach.

\section{Maximalist Approach}

Both a minimalist approach and the Classic and Innovative Goals (a)-(b) lead to a maximalist approach: there should be a philosophy of information understood not merely in the minimalist sense, but above all as a philosophy of information design, which will provide the systematic treatment of the philosophical foundations of an information society, and hence explain and guide the purposeful construction of our intellectual environment. PI is here interpreted as a philosophy of the information environments and therefore as the most fundamental area of philosophical interest in our time. It enables us to make sense of our world and construct it 